\title{
A nomogram for predicting brain metastases of EGFR-mutated lung adenocarcinoma patients and estimating the efficacy of therapeutic strategies
}

\author{
Jing Wang", Baozhong Zhang", Qingsong Pang, Tian Zhang, Xi Chen, Puchun Er, Yuwen Wang, \\ Jinqiang You, Ping Wang
}

Department of Radiation Oncology, Tianjin Medical University Cancer Institute and Hospital, Key Laboratory of Cancer Prevention and Therapy, National Clinical Research Center for Cancer, Tianjin's Clinical Research Centre for Cancer, Tianjin, China

Contributions: (I) Conception and design: J Wang, B Zhang (II) Administrative support: Q Pang; (III) Provision of study materials or patients: P Wang, J You; (IV) Collection and assembly of data: T Zhang, X Chen; (V) Data analysis and interpretation: P Er, Y Wang; (VI) Manuscript writing: All authors; (VII) Final approval of manuscript: All authors.

"These authors contributed equally to this work.

Correspondence to: Jinqiang You; Ping Wang. Department of Radiation Oncology, Tianjin Medical University Cancer Institute and Hospital, Key Laboratory of Cancer Prevention and Therapy, National Clinical Research Center for Cancer, Tianjin's Clinical Research Centre for Cancer, HuanHu-Xi Road, Ti-Yuan-Bei, He Xi District, Tianjin 300060, China. Email: youjq2005@163.com; wangping@tjmuch.com.

Background: To establish a nomogram for predicting the outcome of EGFR-mutated lung adenocarcinoma patients with brain metastases (BMs) and to estimate the efficacy of different therapeutic strategies.

Methods: The data of 129 cases with BM from the period between January 1st 2011 and December 31st 2014 were collected, and all of the cases were pathologically confirmed to be lung adenocarcinoma, stages IIV and with 19 and/or 21 exon mutations of EGFR. Cox regression analysis and log-rank test were used for data analysis. The nomogram was used to establish the progression models.

Results: In the univariate analysis, the stage, ECOG score, interval between the diagnosis of lung cancer and $\mathrm{BM}$, the number of brain metastatic lesions, and the diameter of the maximal brain metastatic lesion correlated well with overall survival (OS). In multivariate Cox proportional hazard analysis, the ECOG score, interval between the diagnosis of lung cancer and BM, and the number of brain metastatic lesions correlated well with the OS. Patients were divided into the poor prognostic group and the good prognostic group based on the nomogram prognostic model score. Subgroup analysis showed that in the poor prognostic group, the OS of patients who received radiotherapy was better than that of the patients who did not receive radiotherapy as the first-line treatment (30 vs. 19 months, $\mathrm{P}<0.05)$. The OS was 30 months in the TKI subgroup and 21 months in the no TKI subgroup, but no statistical difference was found $(\mathrm{P}>0.05)$. Patients in the good prognostic group who received radiotherapy had a better 3-y OS rate than the patients who received no radiotherapy as the first-line treatment $(91.2 \%$ vs. $58.1 \%, \mathrm{P}<0.05)$. The $3-\mathrm{y}$ OS rate was $87.6 \%$ in the TKI subgroup and $67.8 \%$ in the no TKI group $(\mathrm{P}<0.05)$.

Conclusions: We established an effective nomogram model to predict the progression of EGFR-mutated lung adenocarcinoma patients with BM and the therapeutic effect of the individual treatments. Radiotherapy was beneficial for the patients of both the poor and good prognostic groups, but TKI may be better suited for treating the patients with good prognosis.

Keywords: Non-small cell lung cancer (NSCLC); brain metastasis (BM); EGFR mutation; nomogram; prognosis; treatment

Submitted Apr 13, 2020. Accepted for publication Dec 13, 2020.

doi: $10.21037 /$ jtd-20-1587

View this article at: http://dx.doi.org/10.21037/jtd-20-1587 


\section{Introduction}

Lung cancer, a type of malignant tumor, is known the world over for having the highest morbidity and mortality of all cancers. Accounting for $80-85 \%$ of lung cancer cases is non-small cell lung cancer (NSCLC) (1). Brain metastases (BMs) are the main form of distant metastases in lung cancer and one of the common reasons for treatment failure. Approximately $25 \%$ of patients with NSCLC suffer from BM, which influences their survival. Tailored individual survival estimates could prove useful for advising patients with BM on treatmentrelated decisions and enhancing approaches to therapy. In early 1997, RTOG put forward the classification of RPA (recursive partitioning analysis) (2), which was the first prognostic scoring system for assessing the prognosis of patients with BM. In 2008, Sperduto et al. (3) published the Graded Prognostic Assessment (GPA), a new prognostic score system. Individual cancer prognoses are regularly estimated using nomograms (4-9), in the main as they are able to transform statistical predictive models into numerical forms to produce an estimation for the probability of death, recurrence, or another event, taking into consideration the individual's profile (10). Although nomograms are widely used for cancer prognosis (4-9), this approach has not been applied to EGFR-mutated lung adenocarcinoma patients with BM.

NSCLC patients with BMs have poor prognosis despite therapies, with a 1-year survival rate of less than $20 \%$ (3). WBRT (whole-brain radiation therapy) serves as a standard treatment for NSCLC patients with BM, which has resulted in an OS in the range of 3 to 6 months since the 1970s (11,12). Tyrosine kinase inhibitor (TKI), a small molecule with a good lipid-water partition coefficient, can be absorbed with little difficulty and has high permeability; therefore, it is able to pass through the cell membrane and the BBB (blood brain barrier). EGFR mutations are key targets in predicting TKI treatment efficiency for NSCLC patients. More recently, TKI therapy has been explored for brain metastatic patients with EGFR mutations with an effective rate of approximately $70-80 \%$ (13). A Japanese research team reported that among 41 patients, the complete and partial response rate was $87.8 \%$ (14). Furthermore, the intracranial progression free survival time was 14.5 months and the overall survival (OS) was 21.9 months. For almost half of the subjects, radiation therapy was delayed by TKI for over 1.5 years after being diagnosed with BM (14). Accordingly, some experts pointed out that TKI targeted therapy has become a favorable treatment, especially for patients with EGFR mutation of BMs of lung cancer, challenging the status of radiotherapy as a result. However, for individuals with EGFR-mutated NSCLC who have BM, the optimal treatment is yet to be established (15), and this important question remains to be examined.

This study was conducted with the purpose of developing a nomogram model to estimate survival probabilities for individual patients with EGFR-mutated lung adenocarcinoma with BM. We developed a nomogram based on clinical features for predicting prognosis and the value of radiotherapy and TKI for treating the patients with different prognosis.

We present the following article in accordance with the STROBE reporting checklist (available at http://dx.doi. org/10.21037/jtd-20-1587).

\section{Methods}

\section{Study design and patient eligibility}

We collected data from patients who had stage I-IV lung cancer treated at our hospital from January 1st 2011 to December 31st 2014. Patients were included in this study if they were pathologically confirmed to have adenocarcinoma and genetically confirmed to have EGFR mutations in exon 19 and/or exon 21.The patients with meningeal metastases were excluded because of the poor prognosis.

A total of number of 560 cases were initially selected. We analyzed and summarized the 129 (23.0\%) cases who had brain parenchymal metastases at initial diagnosis or developed BMs during routine follow-up as the first progression. The patients were followed up by hospitalization and/or outpatient clinic consultations. The study was conducted in accordance with the Declaration of Helsinki (as revised in 2013). The study was approved by the independent Ethics Committee of Tianjin Medical University Cancer Institute \& Hospital and individual consent for this retrospective analysis was waived.

\section{Treatment schedule}

Diagnosis of BM was made based on an enhanced head MRI. After the diagnosis of BM, 106 (82.3\%) patients received treatments including radiotherapy, chemotherapy, and targeted therapy. Radiotherapy included whole brain radiotherapy (WBRT, 40 Gy/20 f or $30 \mathrm{~Gy} / 10 \mathrm{f}$ ) and/or 
Table 1 Clinical characteristics $(n=129)$

\begin{tabular}{|c|c|}
\hline Factors & $\mathrm{N}(\%)$ \\
\hline \multicolumn{2}{|l|}{ Sex } \\
\hline Male & $50(38.9)$ \\
\hline Female & $79(61.1)$ \\
\hline \multicolumn{2}{|l|}{ Age (years) } \\
\hline$\leq 65$ & $100(80.5)$ \\
\hline$>65$ & $29(19.5)$ \\
\hline \multicolumn{2}{|l|}{ Smoking } \\
\hline Yes & $47(36.4)$ \\
\hline No & $82(63.6)$ \\
\hline \multicolumn{2}{|l|}{ Stage } \\
\hline I-III stage & $110(85.3)$ \\
\hline IV stage & $19(14.7)$ \\
\hline \multicolumn{2}{|l|}{ Mutation type } \\
\hline Exon 19 & $54(41.9)$ \\
\hline Exon 21 & $75(58.1)$ \\
\hline \multicolumn{2}{|c|}{ Control of primary lung cancer } \\
\hline Controlled & $100(77.5)$ \\
\hline Progressive & $29(22.5)$ \\
\hline \multicolumn{2}{|c|}{ Presence of combined extracranial metastasis } \\
\hline Absent & $56(43.4)$ \\
\hline Present & $73(56.6)$ \\
\hline \multicolumn{2}{|c|}{ Occurrence of brain metastases (months) } \\
\hline$\leq 16$ & $71(55.0)$ \\
\hline$>16$ & $58(45.0)$ \\
\hline \multicolumn{2}{|c|}{ Number of brain metastases } \\
\hline$\leq 3$ & $65(50.4)$ \\
\hline$>3$ & $64(49.6)$ \\
\hline \multicolumn{2}{|c|}{ Maximum size of brain metastases $(\mathrm{cm})$} \\
\hline$\leq 2$ & $87(67.4)$ \\
\hline$>2$ & $42(32.6)$ \\
\hline \multicolumn{2}{|c|}{ Symptoms associated with brain metastasis } \\
\hline None & $48(37.2)$ \\
\hline Yes & $81(62.8)$ \\
\hline \multicolumn{2}{|l|}{ ECOG score } \\
\hline$\leq 2$ & $90(69.8)$ \\
\hline$>2$ & $39(30.2)$ \\
\hline \multicolumn{2}{|l|}{$\mathrm{CEA}(\mu \mathrm{g} / \mathrm{mL})$} \\
\hline$\leq 10$ & $86(66.7)$ \\
\hline$>10$ & 43 (33.3) \\
\hline
\end{tabular}

stereotactic radiotherapy. Cisplatin-based chemotherapy was taken by the chemotherapy group. The targeted therapy treatment medicines were Gefitinib $(250 \mathrm{mg}$, oral, once per day), Erlotinib (150 mg, oral, once per day), or Icotinib (125 mg, oral, 3 times per day). The oral medicines were taken until the disease progressed, or an unacceptable adverse reaction or death occurred.

\section{Statistical analyses}

OS was defined as the period of time from diagnosis to death or last follow-up. The Kaplan-Meier method was applied to conduct survival analysis, and log-rank test was used to calculate comparisons between groups. Univariate and multivariate analyses were carried out using Cox proportional hazard models. Statistical significance was considered to exist when $\mathrm{P}$ value $<0.05$. The nomogram was constructed based on the Cox proportional hazards regression model for survival data. The nomogram score was verified by way of receiver operating characteristic (ROC) curves and internal calibration blots. Kaplan-Meier and Cox analysis were carried out using SPSS 18.0 software; and $\mathrm{R}$ software, version 3.2.2, was used for the nomogram, $\mathrm{ROC}$, and internal validation.

\section{Results}

\section{Patient characteristics}

Of the included cases, 50 cases were male (38.9\%) and 79 cases were female (61.1\%). The median age of onset was 58 , and $80.5 \%$ of the patients were $\leq 65$ years. Approximately $36 \%$ of the cases were smokers. Regarding mutations, approximately $42 \%$ of the cases carried exon 19 mutation and $58.0 \%$ of the cases carried exon 21 mutation. The number of patients with stages I-III and stage IV lung adenocarcinoma at initial diagnosis were $110(85.3 \%)$ and 19 (14.7\%), respectively (Table 1).

\section{Kaplan-Meier curves}

The median OS of all patients was 36 months, and the 1 - and 2 -year survival rates were $91.5 \%$ and $72.4 \%$, respectively. The median OS after BM diagnosis was 16 months, and the 1 - and 2-year survival rates were $55.1 \%$ and $46.5 \%$, respectively.

The median follow-up time for the whole group was 28 months, and the follow-up rate was $100 \%$. 
Table 2 Univariate and multivariate Cox proportional hazard regression analysis between clinical variables and OS

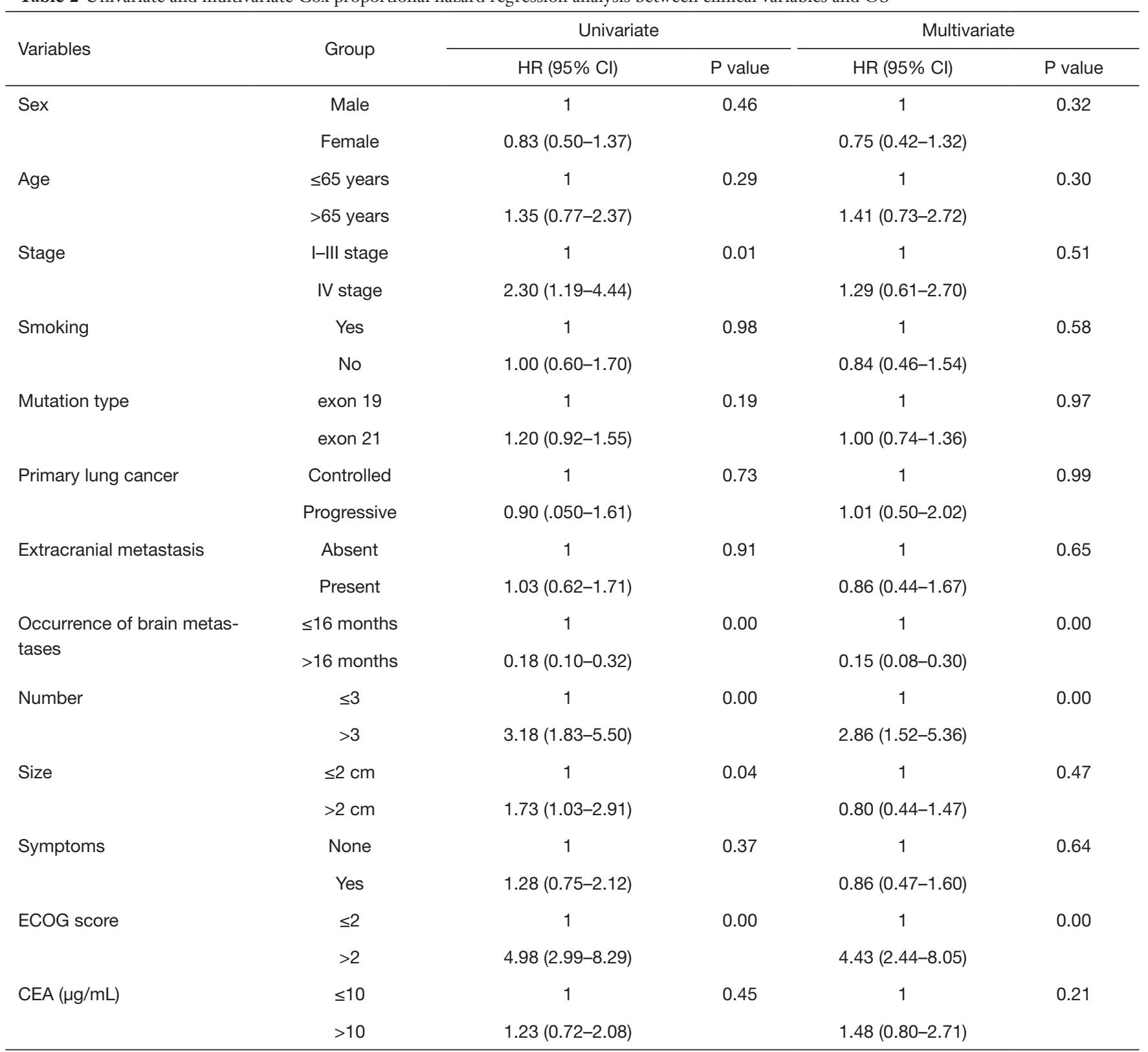

\section{Univariate and multivariate results}

In the univariate analysis, the stage, ECOG score, interval between the diagnoses of lung cancer and $\mathrm{BM}$, the number of brain metastatic lesions, and the diameter of the maximal brain metastatic lesion correlated well with OS. In the multivariate Cox proportional hazard analysis, the ECOG score, interval between the diagnoses of lung cancer and $\mathrm{BM}$, and the number of brain metastatic lesions were the significant prognostic factors related to OS (Table 2).

\section{Nomogram}

The nomogram we developed was based on the significant risk factors $(\mathrm{P}<0.05)$ identified using multivariate analysis. Stage had $\mathrm{P}<0.01$ in the univariate analysis, and it was also taken into account in the nomogram. The nomogram for predicting OS was built based on stage, ECOG score, interval between the confirmed diagnoses of lung cancer and $\mathrm{BM}$, and the number of brain metastatic lesions (Figure 1). The nomogram was used make predictions 


Points
Number
Stage
Interval
Total Points
Linear Predictor
2-year Survival Probability
3-year Survival Probability
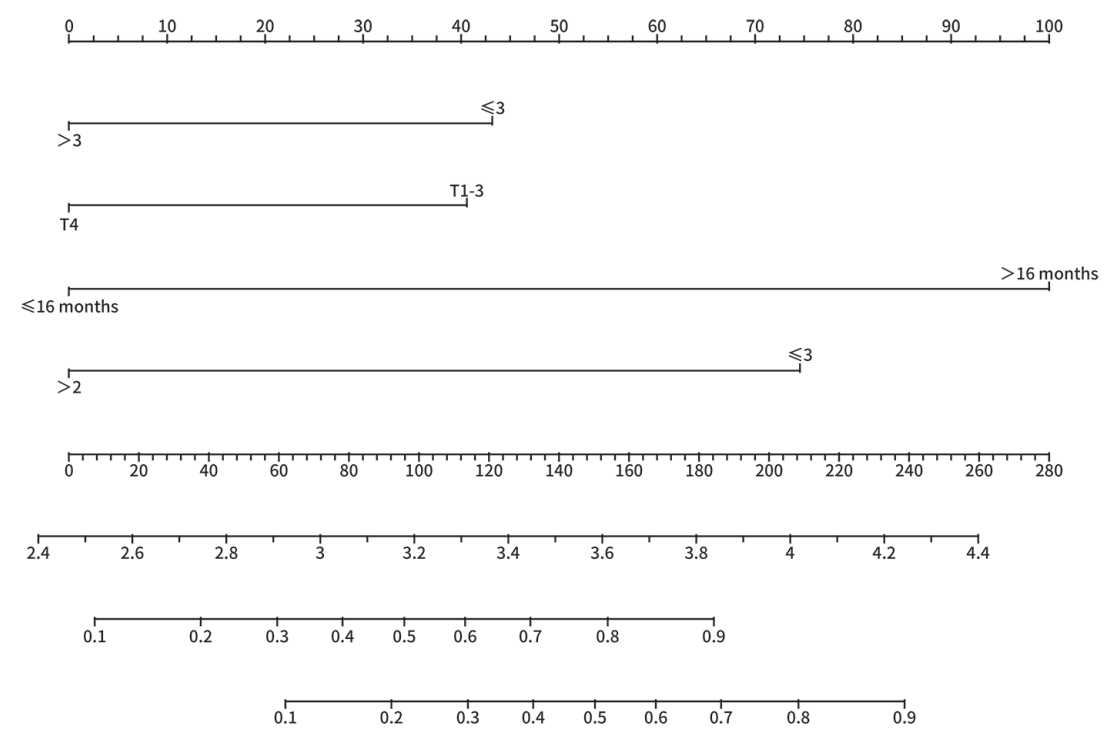

Figure 1 The nomogram for predicting OS was constructed based on the number of metastatic brain lesions, stage, the interval between the confirmed diagnoses of lung cancer and BM, and the ECOG score. OS, overall survival; BM, brain metastasis.
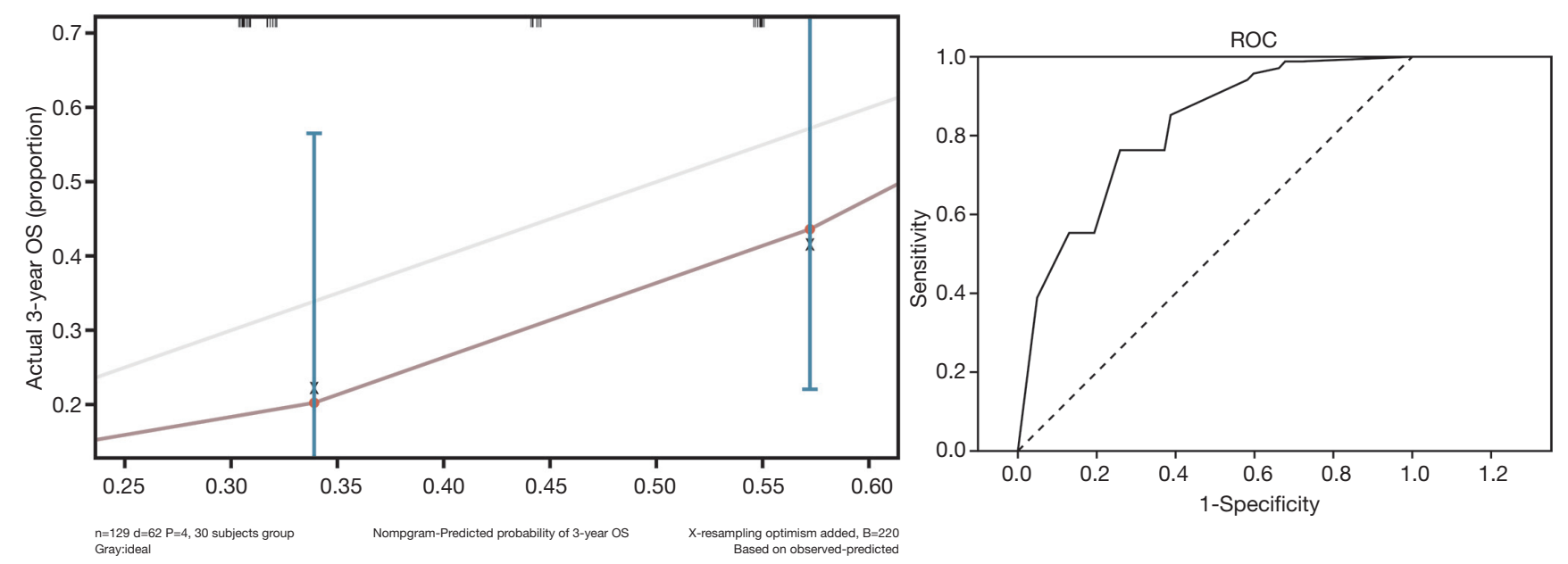

Figure 2 Receiver operating characteristic (ROC) curve and internal calibration plot. The concordance index for the model was 0.83 .

of the 2 - and 3-year OS probabilities; Figure 2 displays associated ROC curves and internal calibration plots. The concordance index for the model was 0.83 . The nomogrampredicted survival was well calibrated with the KaplanMeier observed survival.

\section{Nomogram scores to predict the efficacy in the subgroup analysis}

Risk scores for the patients were calculated based on the nomogram model. The median score of the nomogram was 157 [0-257]. Based on ROC analysis, a cutoff value of 148 was chosen for OS prediction, and the patients were split into two groups, the poor prognostic group (score: $0-148$ ) and the good prognostic group (score $>148$ ) based on this cutoff value. Kaplan-Meier analysis revealed that median OS were 24 months in poor prognostic group and 57 months in good prognostic group $(\mathrm{P}<0.05)$. The $1-$ and 2 -year survival rates in the poor prognostic group were $82.3 \%$ and $49.4 \%$, respectively. The 1 - and 2 -year survival 


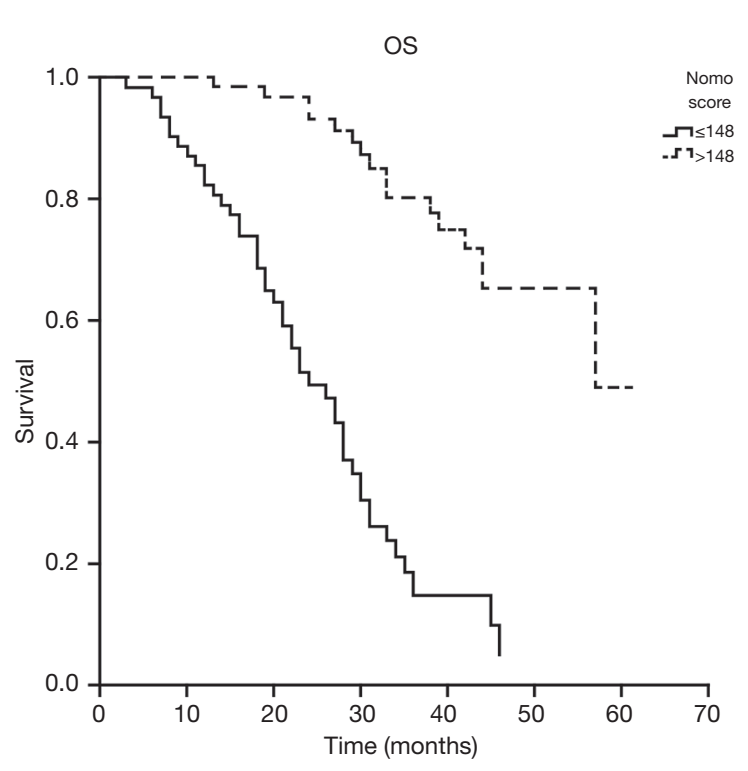

Figure 3 Kaplan-Meier analysis revealed that the median OS was 24 months in the poor prognostic group and 57 months in the good prognostic group $(\mathrm{P}<0.05)$. The 1 - and 2 -year survival rates in the poor prognostic group were $82.3 \%$ and $49.4 \%$, respectively. The 1- and 2-year survival rates in the good prognostic group were $100 \%$ and $93.2 \%$, respectively $(\mathrm{P}<0.05)$. OS, overall survival.

rates for the good prognostic group were $100 \%$ and $93.2 \%$, respectively $(\mathrm{P}<0.05)$ (Figure 3).

Subgroup K-M analysis was conducted to compare the two treatments: radiotherapy and TKI. In the poor prognostic group, patients who received radiotherapy have longer OS than the patients who received no radiotherapy for the first-line treatment ( 30 vs. 19 months, $\mathrm{P}<0.05)$. The OS was 30 months in TKI subgroup and 21 months in no TKI group, which has no statistical difference $(\mathrm{P}>0.05)$. In good prognostic group, patients who received radiotherapy have a higher 3-y OS rate than the patients who received no radiotherapy for the first-line treatment $(91.2 \%$ vs. $58.1 \%$, $\mathrm{P}<0.05$ ). The 3 -y OS rate was $87.6 \%$ in TKI subgroup and $67.8 \%$ in no TKI group, which has a statistical difference $(\mathrm{P}<0.05)$ (Figure 4).

\section{Discussion}

Many patients with lung cancer develop BMs, which impacts quality of life and dramatically decreases survival. Up to $40 \%$ of those who receive an NSCLC diagnosis will go on to develop BMs at some point over the course of their disease; a risk which could be even greater for those who harbor EGFR exon mutations (15). Previous studies have found EGFR mutations to be significantly associated with increased risk of $\mathrm{BM}(16,17)$ and the distinct clinical profiles of EGFR-mutated tumors in relation to BM have been pointed out (18-21). Therefore, it is speculated that $\mathrm{BMs}$ in these patients exhibit their own characteristics in occurrence, treatment, and prognosis.

The results of our univariate analysis showed stage, ECOG score, interval between the diagnoses of lung cancer and $\mathrm{BM}$, and the number and diameter of BMs lesions to be correlated with OS. The multivariate Cox proportional hazard analysis showed that the ECOG score, interval between the confirmed diagnoses of lung cancer and $\mathrm{BM}$, and the number of BMs lesion were the prognostic factors significantly associated with OS. Previous studies concluded that the performance status $(2,11,22,23)$, age $(2,11,22,23)$, extracranial metastases $(2,11,22,23)$, and primary tumor control $(11,16)$ have a potential effect on survival. Other studies $(24,25)$ indicated that the number of brain metastases influences survival. None of the previous studies were targeted on patients with BMs with EGFRmutated lung adenocarcinoma. Most of the patients in our study were treated with TKI, which could have potentially impacted our results that differed from previous studies.

When compared with traditional prognostic indicators, nomograms may provide prognostic estimates which are tailored to individual patients to a greater degree. The predictive and prognostic models were subjected to internal validation, and they were indicated by calibration and discrimination tests to perform well. Previous studies were focused on the patients with BM (26-29). Our study is the first to develop a nomogram for patients with BM with EGFR-mutated lung adenocarcinoma. Our KaplanMeier analysis revealed that the median OS and the 1- and 2 -year survival rates in the high nomogram group were better than in the low nomogram group $(\mathrm{P}<0.05)$. As such, the nomogram may prove useful for assessing risk as well as selecting therapies according to the patient as an individual.

In the past, surgical resection, stereotactic radiosurgery (SRS), or whole-brain radiotherapy, either individually or in combination, have been employed to treat patients with BMs. Tyrosine kinase inhibitor (TKI), a small molecule with a good lipid-water partition coefficient, can be absorbed with little difficulty and has high permeability; therefore, it is able to pass through the cell membrane and the BBB. Some experts have pointed out that TKI targeted therapy is becoming a favorable treatment, especially for patients with EGFR-mutated lung cancer who have of BMs 
A

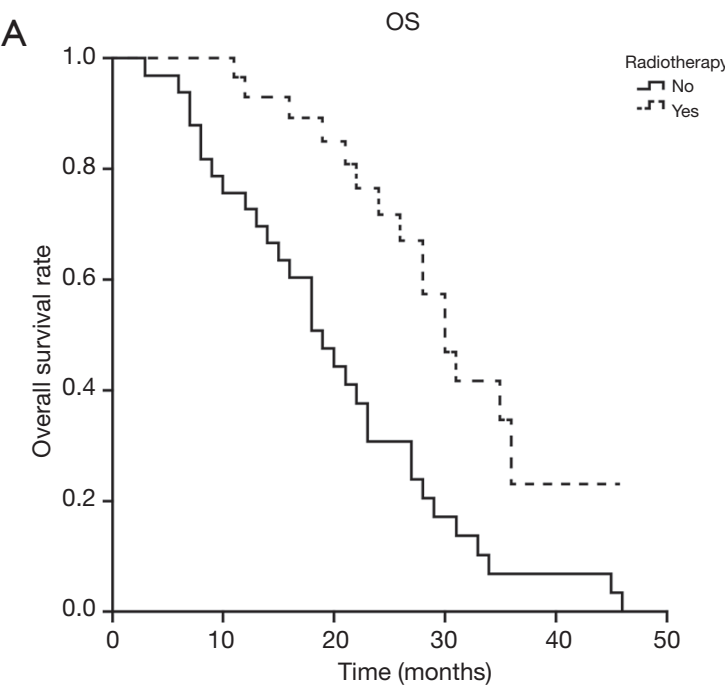

C

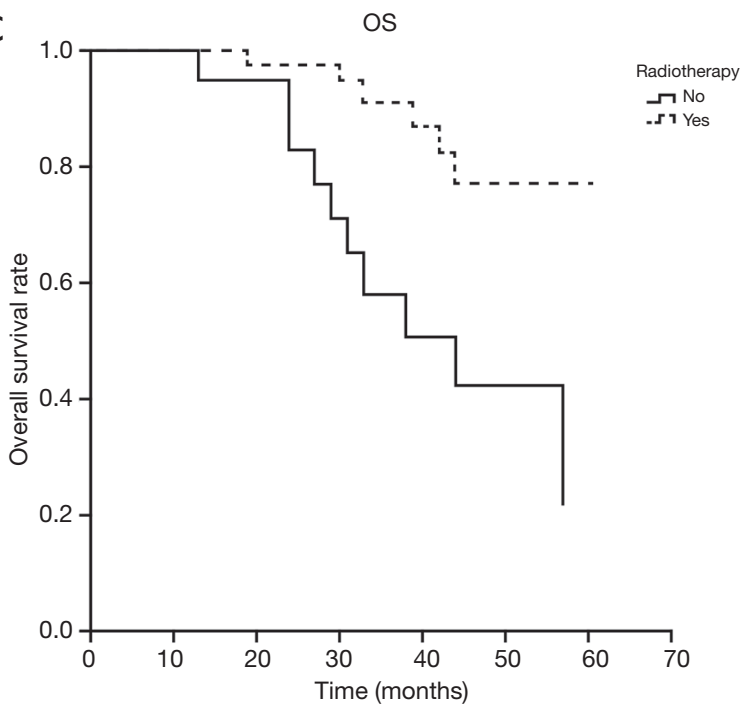

B

B 1.0

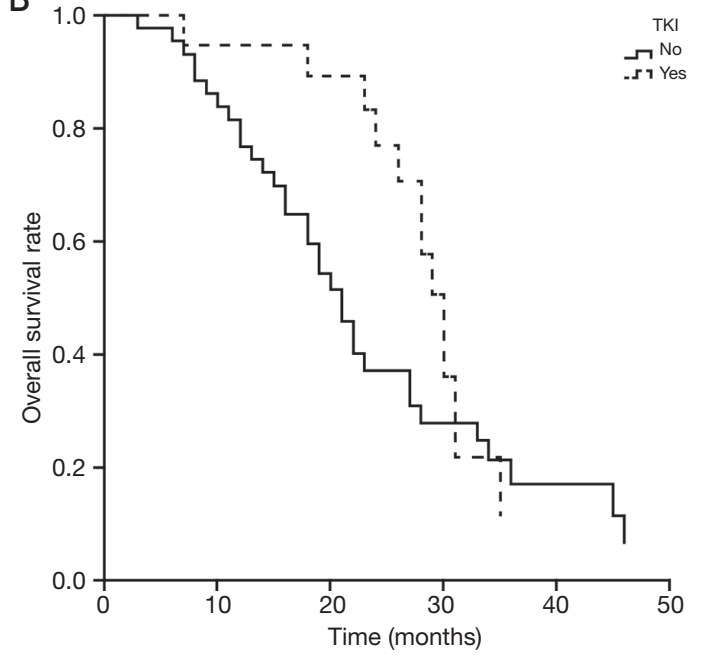

os

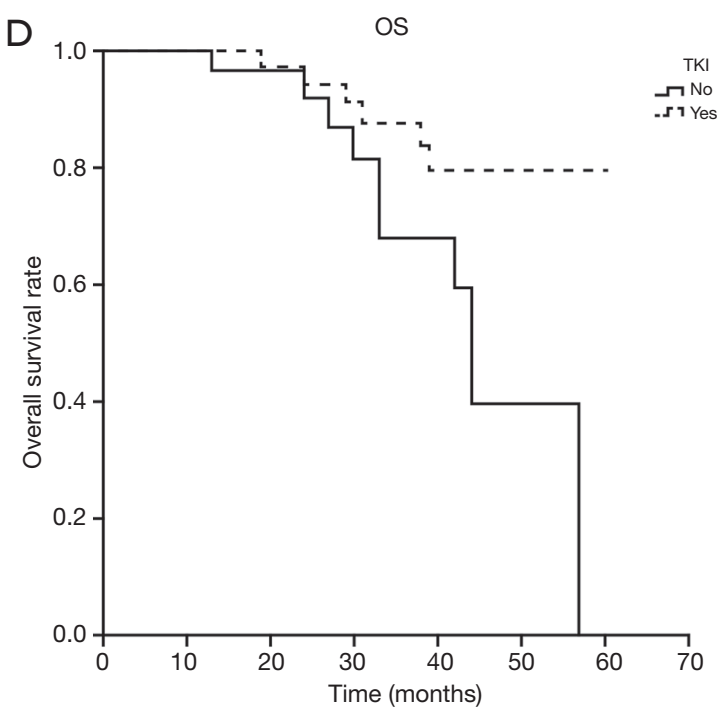

Figure 4 Kaplan-Meier subgroup analysis was conducted to compare the two treatments: radiotherapy and TKI. In the poor prognostic group, patients who received radiotherapy had a better OS than the patients who did not receive radiotherapy as the first-line treatment (30 vs. 19 months, $\mathrm{P}<0.05$, A). The OS was 30 months in the TKI subgroup and 21 months in the no TKI group, which had no statistical significance $(\mathrm{P}>0.05, \mathrm{~B})$. In good prognostic group, patients who received radiotherapy have a higher 3 -y OS rate than the patients who received no radiotherapy for the first-line treatment (91.2\% vs. 58.1\%, $\mathrm{P}<0.05$, C). The $3-\mathrm{y}$ OS rate was $87.6 \%$ in TKI subgroup and $67.8 \%$ in no TKI group, which has a statistical difference $(\mathrm{P}<0.05, \mathrm{D})$. TKI, tyrosine kinase inhibitor; OS, overall survival.

$(13,14)$, while others $(30,31)$ believed that radiotherapy is irreplaceable for these patients. A meta-analysis showed that EGFR-TKIs used by itself should be the first-choice treatment for NSCLC patients who have multiple BM, particularly those with EGFR mutation, as it offers similar OS and extracranial PFS but superior intracranial PFS in comparison with WBRT plus EGFR-TKIs (31). A recent study suggested that in a selective patient group, SRS and TKI could offer, when used in combination, an effective form of treatment for those with BM who have favorable brain control and little neurotoxicity (32). A new study showed that for patients with EGFR-mutated NSCLC who develop BMs, the upfront administration of EGFRTKI and deferral of radiotherapy are related to poorer OS. 
EGFR-TKI following SRS saw the longest OS for patients, allowing them to evade the potential neurocognitive sequelae of WBRT (33). In conclusion, the optimal treatment for EGFR-mutated NSCLC patients with BM 2 is unknown, and this is an important research issue which needs to be addressed. Patients who have different prognoses may require different individualized approaches to treatment. Our research divided patients into two groups, the poor prognostic group and the good prognostic group. Our research indicated that in poor prognostic group, patients who received radiotherapy had a longer OS than the patients who did not receive radiotherapy as the firstline treatment ( $30 v s .19$ months, $\mathrm{P}<0.05)$. The OS was 30 months in the TKI subgroup and 21 months in the no TKI group, and there was no statistical significance $(\mathrm{P}>0.05)$. In the good prognostic group, patients who received radiotherapy had a better 3 -y OS rate than the patients who did not receive radiotherapy as the first-line treatment (91.2\% vs. $58.1 \%, \mathrm{P}<0.05)$. The $3-\mathrm{y}$ OS rate was $87.6 \%$ in the TKI subgroup and $67.8 \%$ in the no TKI group, and there was statistical significance $(\mathrm{P}<0.05)$. We then concluded that radiotherapy was beneficial for all patients, whatever their prognosis, but TKI may be more suitable for the patients who had a better prognosis. In the first 24 months after BM, the prognosis of the TKI subgroup looked better than that of the no TKI subgroup in the poor prognostic group, but after 24 months, the OS curve of the TKI subgroup dropped rapidly. Drug resistance is a likely reason for this. Patients who had a poor prognosis may have a poor performance, more or larger brain metastatic lesions, so it was probably lack of salvage treatment if the drug resistance occurs.

\section{Conclusions}

The nomogram was useful for predicting BMs in EGFRmutated lung adenocarcinoma patients and for selecting individualized therapies. Radiotherapy was beneficial for all the patients, whatever the prognosis, but TKI may be better suited for treating patients who have a better prognosis.

\section{Limitations}

There are certain limitations to this study. First, this study is a retrospective analysis focusing on a relatively small amount of cases and a limited follow-up duration. Therefore, there were differences in the primary treatments patients underwent, and these treatments might have affected the survival assessment. This study therefore cannot offer a comprehensive reflection of prognosis of BMs in patients in relation to this. Improved data collection and/or a randomized controlled study are necessary to explore in-depth answers for these research questions.

\section{Acknowledgments}

Funding: None.

\section{Footnote}

Reporting Checklist: The authors have completed the STROBE reporting checklist. Available at http://dx.doi. org/10.21037/jtd-20-1587

Data Sharing Statement: Available at http://dx.doi. org/10.21037/jtd-20-1587

Conflicts of Interest: All authors have completed the ICMJE uniform disclosure form (available at http://dx.doi. org/10.21037/jtd-20-1587). The authors have no conflicts of interest to declare.

Ethical Statement: The authors are accountable for all aspects of the work in ensuring that questions related to the accuracy or integrity of any part of the work are appropriately investigated and resolved. The study was conducted in accordance with the Declaration of Helsinki (as revised in 2013). The study was approved by the independent Ethics Committee of Tianjin Medical University Cancer Institute \& Hospital and individual consent for this retrospective analysis was waived.

Open Access Statement: This is an Open Access article distributed in accordance with the Creative Commons Attribution-NonCommercial-NoDerivs 4.0 International License (CC BY-NC-ND 4.0), which permits the noncommercial replication and distribution of the article with the strict proviso that no changes or edits are made and the original work is properly cited (including links to both the formal publication through the relevant DOI and the license). See: https://creativecommons.org/licenses/by-nc-nd/4.0/.

\section{References}

1. Qin $\mathrm{H}$, Wang C, Jiang $\mathrm{Y}$, et al. Patients with single brain metastasis from non-small cell lung cancer equally benefit 
from stereotactic radiosurgery and surgery: a systematic review. Med Sci Monit 2015;21:144-52.

2. Gaspar L, Scott C, Rotman M, et al. Recursive partitioning analysis(RPA) of prognostic factors in three Radiation Therapy Oncology Group(RTOG) brain metastases trials. Int J Radiat Oncol Biol Phys 1997;37:745-51.

3. Sperduto PW, Berkey B, Gaspar LE, et al. A new prognostic index and comparison to three other indices for patients with brain metastases: an analysis of 1,960 patients in the RTOG database. Int J Radiat Oncol Biol Phys 2008;70:510-4.

4. Liang W, Zhang L, Jiang G, et al. Development and validation of a nomogram for predicting survival in patients with resected non-small-cell cancer. J Clin Oncol 2015;33:861-9.

5. Wang Y, Li J, Xia Y, et al. Prognostic nomogram for intrahepatic cholangiocarcinoma after partial hepatectomy. J Clin Oncol 2013;31:1188-95.

6. Weiser MR, Landmann RG, Kattan MW, et al. Individualized prediction of colon cancer recurrence using a nomogram. J Clin Oncol 2008;26:380-5.

7. Abdollah F, Karnes RJ, Suardi N, et al. Impact of adjuvant radiotherapy on survival of patients with node-positive prostate cancer. J Clin Oncol 2014;32:3939-47.

8. Balachandran VP, Gonen M, Smith JJ, et al. Nomograms in oncology: more than meets the eye. Lancet Oncol 2015;16:e173-80.

9. Van Zee KJ, Patil S. Validation of a nomogram for predicting risk of local recurrence for ductal carcinoma in situ. J Clin Oncol 2012;30:3143-4.

10. Iasonos A, Schraq D, Rai GV, et al. How to build and interpret a nomogram for cancer prognosis. J Clin Oncol 2008;26:1364-70.

11. Diener-West M, Dobbins TW, Phillips TL, et al. Identification of an optimal subgroup for treatment evaluation of patients with brain metastases using RTOG study 7916. Int J Radiat Oncol Biol Phys 1989;16:669-73.

12. Borgelt B, Gelber R, Kramer S, et al. The palliation of brain metastases: final results of the first two studies by the Radiation Therapy Oncology Group. Int J Radiat Oncol Biol Phys 1980;6:1-9.

13. Park SJ, Kim HT, Lee DH, et al. Efficacy of epidermal growth factor receptor tyrosine inhibitors for brain metastases in non-small cell lung cancer patients harboring either exon 19 or 21 mutation. Lung Cancer 2012;77:556-60.

14. Iuchi T, Shingyoji M, Sakaida T, et al. Phase II trial of gefitinib alone without radiation therapy for Japanese patients with brain metastases from EGFR-mutant lung adenocarcinoma. Lung Cancer 2013;82:282-7.

15. Lu Y, Fan Y. Combined action of EGFR tyrosine kinase inhibitors and whole-brain radiotherapy on EGFRmutated non-small-cell lung cancer patients with brain metastases, Onco Targets Ther 2016;9:1135-43.

16. Shin DY, Na II, Kim CH, et al. EGFR mutation and brain metastases in pulmonary adenocarcinoma. J Thorac Oncol 2014;9:195-9.

17. Lee YJ, Park IK, Park MS, et al. Activating mutations within the EGFR kinase domain: a molecular predictor of disease-free survival in resected pulmonary adenocarcinoma. J Cancer Res Clin Oncol 2009;135:1647-54.

18. Iuchi T, Shingyoji M, Itakura $M$, et al. Frequency of brain metastases in non-small cell lung cancer, and their association with epidermal growth factor receptor mutations. Int J Clin Oncol 2015;20:674-9.

19. Heon S, Yeap BY, Britt GJ, et al. Development of central nervous system metastases in patients with advanced non-small cell lung cancer and somatic EGFR mutations treated with gefitinib or erlotinib. Clin Cancer Res 2010;16:5873-82.

20. Eichler AF, Kahle KT, Wang DL, et al. EGFR mutation status and survival; after diagnosis of brain metastases in non small cell lung cancer. Neuro Oncol 2010;12:1193-9.

21. Enomoto Y, Takada K, Hagiwara E, et al. Distinct features of distant metastases and lymph node stage in lung adenocarcinoma patients with epidermal growth factor receptor gene mutations. Respir Investig 2013;51:153-7.

22. Gerosa M, Nicolato A, Foroni R, et al. Analysis of longterm outcomes and prognostic factors in patients with nonsmall cell lung cancer brain metastases treated by gamma knife radiosurgery. J Neurosurg 2005;102 Suppl:75-80.

23. Zindler JD, Rodrigues G, Haasbeek CJ, et al. The clinical utility of prognostic scoring systems in patients with brain metastases treated with radiosurgery. Radiother Oncol 2013;106:370-4.

24. Rades D, Schild SE, Lohynska R, et al. Two radiation regimens and prognostic factors for brain metastases in non-small cell lung cancer patients. Cancer 2007;110:1077-82.

25. Sperduto PW, Kased N, Roberge D, et al. Summary report on the graded prognostic assessment: an accurate and facile diagnosis-specific tool to estimate survival for patients with brain metastases. J Clin Oncol 2012;30:419-25.

26. Barnholtz-Sloan JS, Sloan AE, Davis FG, et al. Incidence proportions of brain metastases in patients diagnosed (1973 
to 2001) in the Metropolitan Detroit. Cancer Surveillance System. J Clin Oncol 2004;22:2865-72.

27. Rotin DL, Paklina OV, Kobiakov GL, et al. Lung cancer metastases to the brain: Clinical and morphological prognostic factors. Zh Vopr Neirokhir Im N N Burdenko 2013;77:24-8.

28. Barnholtz-Sloan JS, Yu C, Sloan AE, et al. A nomogram for individualized estimation of survival among patients with brain metastasis. Neuro Oncol 2012;14:910-8.

29. Park Y, Kim KS, Kim K, et al. Nomogram prediction of survival in patients with brain metastases from hepatocellular carcinoma treated with whole-brain radiotherapy: a multicenter retrospective study. J Neurooncol 2015;125:377-83.

30. Omuro AMP, Kris MG, Miller VA, et al. High incidence of disease recurrence in the brain and leptomeninges in

Cite this article as: Wang J, Zhang B, Pang Q, Zhang T, Chen X, Er P, Wang Y, You J, Wang P. A nomogram for predicting brain metastases of EGFR-mutated lung adenocarcinoma patients and estimating the efficacy of therapeutic strategies. J Thorac Dis 2021;13(2):883-892. doi: 10.21037/jtd-20-1587 patients with non small cell lung carcinoma after response to gefitinib. Cancer 2005;103:2344-8.

31. Zheng H, Liu QX, Hou B, et al. Clinical outcomes of WBRT plus EGFR-TKIs versus WBRT or TKIs alone for the treatment of cerebral metastatic NSCLC patients: a meta-analysis. Oncotarget 2017;8:57356-64.

32. Yang WC, Xiao F, Shih JY, et al. Epidermal growth factor receptor mutation predicts favorable outcomes in nonsmall cell lung cancer patients with brain metastases treated with stereotactic radiosurgery. Radiother Oncol 2018;126:368-74.

33. Magnuson WJ, Lester-Coll NH, Wu AJ, et al. Management of Brain Metastases in Tyrosine Kinase Inhibitor-Naive Epidermal Growth Factor ReceptorMutant Non-Small-Cell Lung Cancer: A Retrospective MultiInstitutional Analysis. J Clin Oncol 2017;35:1070-7. 